\title{
DroMOOC: a Massive Open Online Course on Drones and Aerial Multi Robot Systems
}

\author{
Sylvain Bertrand, Julien Marzat \\ ONERA-The French Aerospace Lab \\ Université Paris-Saclay \\ Palaiseau, France \\ sylvain.bertrand@onera.fr \\ julien.marzat@onera.fr
}

\author{
Cristina Stoica Maniu, Maria Makarov \\ Laboratoire des Signaux et Systèmes (L2S) \\ CentraleSupélec-CNRS-Université Paris-Sud \\ Université Paris-Saclay \\ 3 rue Joliot Curie, Gif sur Yvette, France \\ cristina.maniu@centralesupelec.fr \\ maria.makarov@centralesupelec.fr
}

\author{
David Filliat, Antoine Manzanera \\ ENSTA-ParisTech/U2IS \\ Palaiseau, France \\ david.filliat@ensta-paristech.fr \\ antoine.manzanera@ensta-paristech.fr
}

\section{INTRODUCTION}

An important increase of the number of jobs is expected in the next few years on markets related to Unmanned Aerial Vehicles (UAVs). Therefore educational curricula in this field of expertise are subject to more and more demands from students. In addition and complement to traditional courses, Massive Open Online Courses (MOOCs) are also getting a growing interest from students.

This new MOOC, named DroMOOC, is dedicated to drones and aerial Multi Robot Systems (MRS). It aims at covering all the methods that are required to make an aerial vehicle autonomous and several of them to cooperate. It is realized by research scientists, engineers and teachers from three French research and education institutions: ONERA - the French AerospaceLab, CentraleSupélec/Laboratory of Signals and Systems and ENSTA - two graduate engineering schools.

There already exist some MOOCs related to these topics (e.g. see [1], [2]). This MOOC intends to complete this offer to students with some originality in the pedagogic approach, as presented in the next section.

\section{MAIN CHARACTERISTICS OF THE DROMOOC}

With this MOOC, students will be able to understand the main issues related to robotic aerial platforms. They will learn and develop new skills in this field at two different levels of expertise and complexity. Indeed, each course will be available in a basic and, when it makes sense, in an advanced level.

The basic level presents simple notions that are accessible to students with an elementary scientific background and that can easily be applied in practice to a real drone (e.g. for a student project). This basic level will cover all the topics and is a standalone complete course.

The advanced level addresses more complex methods that are closer to the state of the art of research or R\&D in industry. It aims at giving to the students skills that can be professionally valorized (e.g. for a $\mathrm{PhD}$, a job, etc.) and applied in practice

This e-learning project is funded by AAP IDEX 2017 Paris-Saclay, Numérique pour la formation à distance, in the context of Université Paris Saclay \& Programme d'Investissements d'Avenir from French State and ANR. to a real robot leading to improved performances compared to the basic level.

Theoretical background will be reinforced by some practical experience to be valorized for the students for professional perspectives. In robotics, it is mandatory for the students to master some programming languages and tools such as Python and ROS. Therefore, such practical aspects are considered in this MOOC. A virtual machine running Linux Ubuntu and ROS can be downloaded by the students. It gathers a set of codes, scripts and experimental data sets that can be easily used as a complement to the video contents of the courses. Experimental data sets are created using the facilities of the COPERNIC Lab of ONERA [3] which disposes of several drones and motion capture system that can be used for example to provide a ground truth associated to the experimental data.

A third particularity of this MOOCs is that it aims at addressing both mono and multi aerial robot systems.

\section{Content of the Dromooc}

The following topics are addressed in this MOOC:

- Aerial mono robots: UAV dynamics modeling, sensor fusion and state estimation, computer vision, environment modeling, SLAM, trajectory planing, control.

- Aerial multi robot systems: multi robot systems modeling, localization, SLAM, cooperative control.

Courses for most of these topics are available in both the basic and advanced levels. A specific course is also dedicated to the use of ROS and the virtual machine.

A validation test, under the form of multiple choice questions and answers, is proposed for each module of the course. A certificate will be delivered to the students with their score.

\section{CONCLUSION}

The MOOC will be freely available online late 2018 at the following URL: www.onera.fr/dromooc.

\section{REFERENCES}

[1] Univ. of Pennsylvania, "Robotics", MOOC available on COURSERA.

[2] Technishe Univ. München, "Autonomous Navigation for Flying Robots", MOOC available on EDX.

[3] www.onera.fr/copernic 